\title{
Article
}

\section{Design of Single Phase SiC Bidirectional DC-AC Converter with Low-Cost PLL for Power Factor Correction}

\author{
Alexandre Bento ${ }^{1, *}$, Sérgio André ${ }^{1}$, Ricardo Luís ${ }^{1,2}$ and J. Fernando Silva ${ }^{2}$ \\ 1 Instituto Superior de Engenharia de Lisboa, Polytechnic Institute of Lisbon, 1959-007, Portugal; \\ sergio.andre1992@gmail.com; rluis@deea.isel.pt \\ 2 INESC-ID, Instituto Superior Técnico, Universidade de Lisboa, 1049-001, Portugal; \\ fernando.alves@tecnico.ulisboa.pt \\ * Correspondence: alexandre-bento@outlook.com; Tel.: +351-21-831-7273
}

\begin{abstract}
The paper presents the design stages of a single-phase Silicon Carbide bidirectional DC-AC converter. This includes the LCL filter design responsible to meet grid connection requirements. A $3 \mathrm{~kW}$ laboratory prototype of the power converter is built employing a low-cost phase locked loop and its results are presented. The design of the low-cost phase locked loop and its implementation are depicted in some detail.
\end{abstract}

Keywords: SiC bidirectional AC-DC converter; inverter; variable frequency; PLL; LCL filter

\section{Introduction}

Nowadays, society has a big interest in topics involving sustainability and energy efficiency mainly due to environmental concerns, [1]. This is observed on the increasing number of electric vehicles and their interaction with the grid, [1]. It is also seen in micro, medium and big scale renewable energy applications with and without energy storage systems, [2]. On a political level, this is shown with various funds and measures aimed at encouraging the investment in these themes. Most of these applications use electronic power converters, which has led to recent developments in the technology of semiconductor devices originating wide band gap semiconductors, [3]. The most promising devices from this group are Silicon Carbide ( $\mathrm{SiC})$ and Gallium Nitrite (GaN) devices. Compared to the traditional Silicon (Si) semiconductors, wide band gap devices support higher current densities, higher switching frequency and higher operation temperatures, $[4,5]$. This contributes to more compact and efficient power converters decreasing the size of passive devices such as inductors and capacitors, [6]. This paper presents a simple design approach for a SiC bidirectional DC-AC converter that permits the charge and discharge of a battery bank to the AC grid with unity power factor used for a grid connected Telecom Energy Storage application. Inductor design, AC filter design and the controller principles are also treated. A $3 \mathrm{~kW}$ converter prototype is implemented in the laboratory and its results presented.

\section{SiC Bidirectional DC-AC Converter Control Principles}

The topology analyzed in this work is a non-isolated bidirectional DC-AC converter, with unity power factor, presented on Figure 1. 


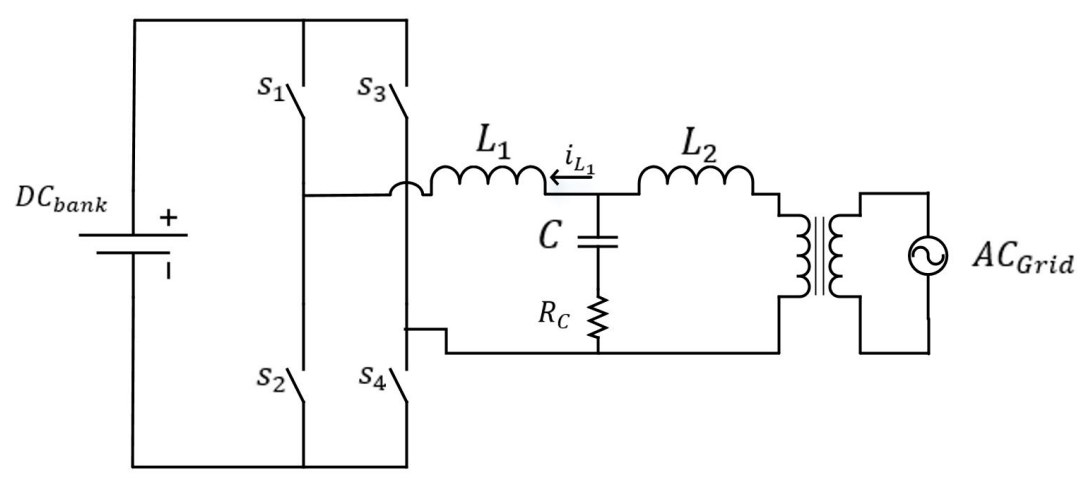

Figure 1. Proposed DC-AC Circuit topology

\subsection{Output LCL Filter}

Grid connected power electronic converters have Power Quality requirements. This is translated into keeping the Total Harmonic Distortion (THD) lower than 5\%, according to IEEE 519-1992 standard. This is accomplished using a filter between the switches and the grid.

The filter has a big influence on the converter's size, cost and performance. It's possible to reduce its size using higher switching frequencies, which is one of the reasons why wide band gap semi-conductors contribute to smaller and more efficient power converters.

On the other side, exploring the switches at their maximum frequency all the time will lead to excess switching losses. For this reason, variable switching frequency is used on this work. This is done by controlling the current on the inductor $L_{1}$ within a hysteresis window, as seen on Figure 2 .

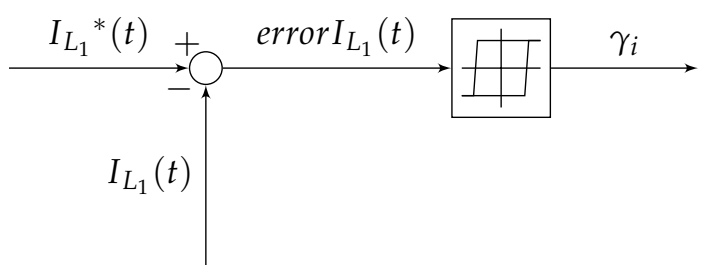

Figure 2. Inverter control block diagram

THD is calculated by (1), where $I_{50 H z}$ is the pretended grid's current and $I_{n^{t h}}$ is is the $n^{\text {th }}$ harmonic of the ripple current which is due to the current hysteresis control window. Although choosing a very small hysteresis error window width would result in a low THD, it would require a very high switching frequency or a very large filter inductor. So there's a trade-off between to minimizing the THD, keeping the switching frequency within feasible values to limit the switching losses and maintaining the inductor with a compact size, [7].

$$
T H D=\frac{\sqrt{\sum_{n=1}^{\infty} I_{n^{t h} s w}^{2}}}{I_{50 H z}}
$$

To summarize, the semiconductor's maximum switching frequency and the need to maintain THD low by keeping the current hysteresis window small obligates a certain inductance value for $L_{1}$.

Since this work is applied for telecom energy storage systems, and this usually happens at low DC voltages (typically 48V), a $3 \mathrm{kVA}$ single phase transformer is used for grid connection. This transformer has a short circuit inductance that combined with the grid's inductance represents the second inductor on the LCL filter, $L_{2}$. 
It's then possible to obtain a 3rd order filter by adding a capacitor, with value $C$, between the inductors and the grid. LCL filter's natural frequency, $\omega_{0}$, is given by (2). It's important to maintain the filter's natural frequency within a tenth of the minimum switching frequency and ten times higher than the grid's frequency $(50 \mathrm{~Hz})$. In order to be stable, this kind of filters need a damping resistance, usually connected in series with inductor, $L_{2}$, or with the capacitor, $C$. Since the current passing to the grid is large in this application, the choice to use the resistance in series with the capacitor is made. The LCL filter transfer function with the capacitor series resistance is given by (3), where $v_{i}$ is the full bridge input pulsed voltage to the filter, $i_{2}$ is the grid's current on the low voltage side of the transformer, $L_{1}, L_{2}, C$ and $R_{C}$ are the filter parameters shown on Figure 1 . These parameters are calculated using (4), (5) and (6). $L_{2}$ is measured, it is the short-circuit impedance of the transformer plus the line inductance seen from the low voltage side of the transformer. Table 1 presents the values used for these calculations during this research work.

$$
\begin{gathered}
\omega_{0}=\sqrt{\frac{L_{1}+L_{2}}{L_{1} \cdot L_{2} \cdot C}} \\
\frac{i_{2}}{v_{i}}=\frac{\frac{s R_{C} C+1}{L_{1} L_{2} C}}{s\left(s^{2}+s \frac{R_{C}\left(L_{1}+L_{2}\right)}{L_{1} L_{2}}+\frac{L_{1}+L_{2}}{L_{1} L_{2} C}\right)} \\
L_{1}=\frac{U_{D C}}{2 \cdot \Delta i_{L_{1}} \cdot f_{s w}} \\
C=\frac{L_{1}+L_{2}}{L_{1} L_{2} \omega_{0}^{2}} \\
R_{C}=2 \cdot \zeta \cdot \omega_{0} \cdot \frac{L_{1} L_{2}}{L_{1}+L_{2}}
\end{gathered}
$$

Table 1. Filter Parameters

\begin{tabular}{|c|c|}
\hline Parameter & Value \\
\hline$U_{D C}$ & $64 \mathrm{~V}$ \\
\hline$\Delta i_{L_{1}}$ & $6.8 \mathrm{~A}$ \\
\hline$L_{1}$ & $48 \mu \mathrm{H}$ \\
\hline$L_{2}$ & $32 \mu \mathrm{H}$ \\
\hline$C$ & $220 \mu \mathrm{F}$ \\
\hline$R_{C}$ & $0.45 \Omega$ \\
\hline$\omega_{0}$ & $15386 \mathrm{rad} / \mathrm{s}$ \\
\hline$\zeta$ & $\sqrt{2} / 2$ \\
\hline
\end{tabular}

\subsection{Inductor Design}

Inductors are of great impact on the size, cost and efficiency of a power converter[6][9]. With the improvements in the semiconductors devices, it's of great importance the effects of higher frequency on the inductor losses. The higher the frequency, higher the inductor core and winding losses[10]. Power inductors aren't exactly off-the-shelf components, each system has its own needs. In this section, it's presented the power inductor design procedure for a prototype application.

The power inductor design starts with the choice of the core material. This should be addressed carefully since there are several materials available and all have their pros and cons, the right choice depends on the design priorities and specifications, [11]. Core suppliers for each specific core give the inductance factor. The inductance is then calculated using (7), where $N$ is the number of wire turns and $A_{L}$ is the core inductance factor. It's important to note that in cores with gaps, $A_{L}$ needs to be 
adjusted to include the gap effects, also it's important to take into account the core saturation, which leads to a lower value of inductance.

$$
L=N^{2} \cdot A_{L}
$$

There are a number of methods to calculate inductor's core loss, some extremely complex and only applicable for certain conditions, [9]. Core suppliers usually make available a set of curves for each material at specific frequencies, with straightforward formulas that can predict the core losses for applications similar to the one treated in this article.

For power inductors, the high-frequency effects on the windings must be taken into account. These high-frequency effects result in extra losses and are due to the skin and proximity effects that are manifested by changing the current density inside the conductor's section, [10,12].

The total winding losses for the application treated in this work is given by (8), where $R_{50 \mathrm{~Hz}}$ is the wire resistance at $50 \mathrm{~Hz}$ that is very close to the DC resistance, $\mathrm{I}_{50 \mathrm{~Hz}}$ is the pretended $50 \mathrm{~Hz}$ current on the inductor, $R_{n^{t h}}$ is is the wire resistance for the $n^{\text {th }}$ harmonic due to switching frequency, and $I_{n^{t h} s w}$ is the $n^{\text {th }}$ current harmonic due to switching.

$$
P_{\text {winding }}=R_{50 H z} I_{50 H z}^{2}+\sum_{n=1}^{\infty} R_{n^{t h} s w} I_{n^{t h} s w}^{2}
$$

\subsection{Phase Locked Loop}

A Phase Locked Loop (PLL) is a control system that compares an input signal with a signal generated by a voltage controlled oscillator ( $\mathrm{VCO}$ ) to produce a multiplied, divided or a synchronous signal [8]. This circuit is typically used in frequency demodulators and its block diagram configuration is presented on Figure 4, as described in [8]. For this work, the PLL will be implemented in software in order to obtain a low-cost grid synchronization tool to allow the control of active power and an unity power factor.

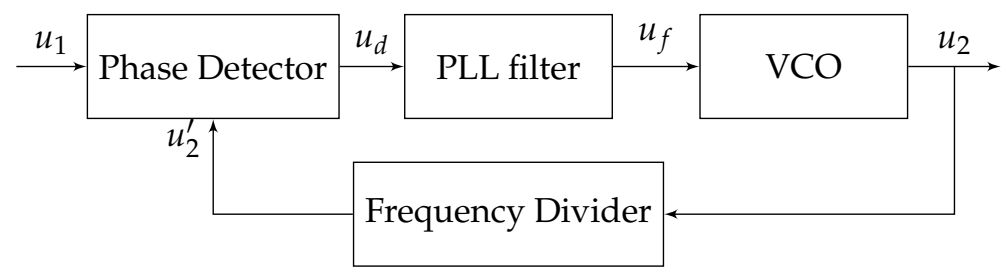

Figure 3. Typical PLL blocks diagram.

As a synchronization application, the frequency divider block isn't applicable since the required input and the output frequencies are the same. This simplification allows Figure 4.

The phase detector block is responsible for giving as output the signal, $u_{d}$, which is proportional to the difference between the phases of the input signal, $u_{1}$, and the feedback signal, $u_{2}$. The PLL filter controls the PLL response. The filter's output signal, $U_{f}$, is integrated by the VCO block generating a ramp that symbolizes the grid's phase angle. This output angle signal is also used to generate a sinusoid wave for feedback. This way it's possible to know exactly the grid's phase angle and produce the reference of current needed to control active and reactive power exchange with the grid.

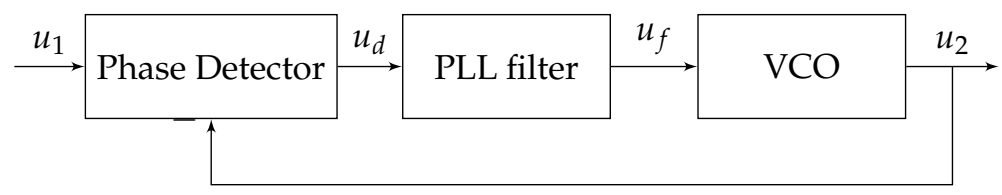

Figure 4. Typical PLL blocks diagram for synchronization applications. 
During the PLL design it is necessary to make the assumption that the PLL is in locked mode, this means that the frequencies of the input signal, $\omega_{1}$, and feedback signal, $\omega_{2}$ are the same. This consideration facilitates the multiplication between the squared input signal, $u_{1}$, and the sinusoidal feedback signal $u_{2}$, given in (9), where $\theta_{1}$ is the input signal's phase angle and $\theta_{2}$ is the feedback signal's phase angle.

$$
u_{d}=\frac{2 \cdot U_{1} \cdot U_{2}}{\pi}\left(\sin \left(\theta_{1}-\theta_{2}\right)+\text { harmonicterms }\right)
$$

Ignoring the harmonics terms and considering the low difference between the angles, when the PLL is in locked mode, is possible to simplify the $\sin \left(\theta_{1}-\theta_{2}\right)$ as $\theta_{1}-\theta_{2}$ (10). This simplification is the linearized model of the phase detector where the inputs are the angles (input and feedback) and not the voltage signals, as shown in Figure 5 [13].

From (10) it's possible to obtain the phase detector block gain, $k_{d}$, given by (11), [13], where $U_{1}$ is the square wave amplitude, and $U_{2}$ is the feedback wave amplitude.

$$
\begin{gathered}
u_{d}=\frac{2 \cdot U_{1} \cdot U_{2}}{\pi}\left(\theta_{1}-\theta_{2}\right) \\
K_{d}=\frac{2 \cdot U_{1} \cdot U_{2}}{\pi}
\end{gathered}
$$

For the filter block, a proportional-integral filter (PI) is chosen because of its pole on origin which improves angle response[13]. The filters block transfer function is given by (12), where $\tau_{1}$ and $\tau_{2}$ are the PI filter parameters. The VCO transfer function is given by (13), being $K_{0}$ the VCO gain.

$$
\begin{gathered}
\frac{U_{f}(s)}{U_{d}(s)}=\frac{1+s \cdot \tau_{2}}{\tau_{1} \cdot s} \\
\frac{K_{0}}{s}
\end{gathered}
$$

The complete PLL block diagram is shown on Figure 5.

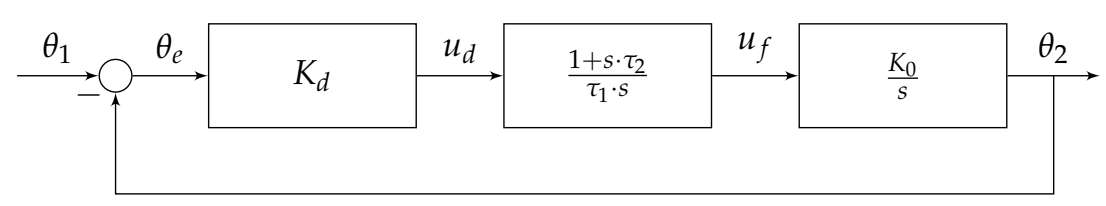

Figure 5. PLL block diagram for design

It's possible to obtain the open loop, $G(s)$, and the closed loop, $\frac{\theta_{o}(s)}{\theta_{i}(s)}$, transfer functions, given respectively by (14) and (15).

$$
\begin{gathered}
G(s)=K_{d} \cdot \frac{1+s \cdot \tau_{2}}{\tau_{1} \cdot s} \cdot \frac{K_{0}}{s}=\frac{\frac{K_{d} \cdot K_{0} \cdot \tau_{2}}{\tau_{1}} \cdot s+\frac{K_{d} \cdot K_{0}}{\tau_{1}}}{s^{2}} \\
\frac{\theta_{o}(s)}{\theta_{i}(s)}=\frac{G(s)}{1+G(s)}=\frac{\frac{K_{d} \cdot K_{0} \cdot \tau_{2}}{\tau_{1}} \cdot s+\frac{K_{d} \cdot K_{0}}{\tau_{1}}}{s^{2}+\frac{K_{d} \cdot K_{0} \cdot \tau_{2}}{\tau_{1}} \cdot s+\frac{K_{d} \cdot K_{0}}{\tau_{1}}}
\end{gathered}
$$

The closed loop transfer function is very similar to a well known equation on systems control theory given by (16), where $\zeta$ is the system damping coefficient and $\omega_{n}$ its natural frequency.

$$
\frac{2 \cdot \zeta \cdot \omega_{n} \cdot s+\omega_{n}^{2}}{s^{2}+2 \cdot \zeta \cdot \omega_{n} \cdot s+\omega_{n}^{2}}
$$

Comparing the terms from (15) to (16) it's possible to make the assumptions given by (17) and
(18). 


$$
\begin{gathered}
\frac{K_{d} \cdot K_{0} \cdot \tau_{2}}{\tau_{1}}=2 \cdot \zeta \cdot \omega_{n} \\
\frac{K_{d} \cdot K_{0}}{\tau_{1}}=\omega_{n}^{2}
\end{gathered}
$$

99 Using $K_{0}=100, \zeta=\frac{\sqrt{2}}{2}, \omega_{n}=\frac{2 \cdot \pi \cdot 50}{10}$ (10 AC grid cycles), it's then calculated $K_{d}, \tau_{1}$ and $\tau_{2}$, see Table 2.

Table 2. PLL design parameters

\begin{tabular}{|c|c|}
\hline Parameter & Value \\
\hline$\zeta$ & 0.707 \\
\hline$\omega_{n}$ & 31.415 \\
\hline$U_{1}$ & 1 \\
\hline$U_{2}$ & 1 \\
\hline$K_{0}$ & 100 \\
\hline$K_{d}$ & 0.637 \\
\hline$\tau_{1}$ & 0.065 \\
\hline$\tau_{2}$ & 0.045 \\
\hline
\end{tabular}

The simulation results obtained using MATLAB®) (The MathWorks Inc., Natick, MA, USA) simulation enviroment are presented on Figure 6.

Results for an input wave with $50 \mathrm{~Hz}$

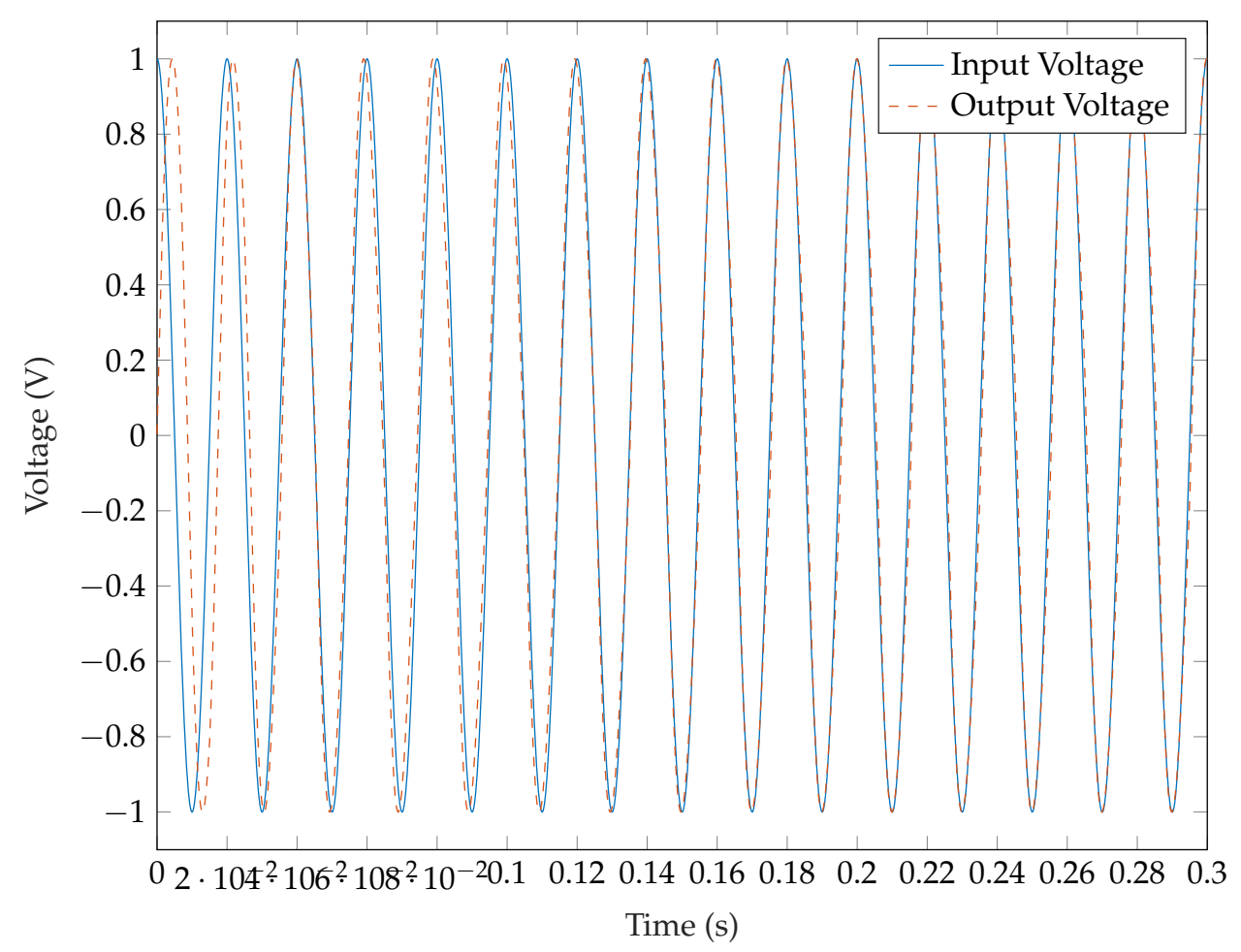

Figure 6. PLL simulation results

The multiplier phase detector has a problem resultant from the multiplication of the two signals (input and feedback), this is the introduction of harmonics in the signal $U_{d}$, given in (9) [13]. In order to attenuate these harmonics, it's typically used a complex or heavy computing algorithm as an orthogonal signal generator [14] or a notch filter [15]. The use of notch filter, in this case, is not 
possible because a notch filter is a very tight band rejection filter, this would only reduce one harmonic component, leaving the others. In the case of an orthogonal signal generator, this option needs a very large floating point variables, and with a low-cost FPGA, the variable size and the number of nodes are limited. Because of this, the final implementation was a 2nd order low-pass filter, presented on Figure 7, implemented with two 1st order filters in cascade to reduce the coefficient variable sizes. This filter is applied to the sine wave generated for reference with a cutoff frequency of $50 \mathrm{~Hz}$ to allow this frequency and block part of the 2 nd harmonic $(100 \mathrm{~Hz})$ and from there on. For convenience, it was implemented an input on the filter's block, $\theta^{*}$, to enable shifting the output sinusoidal wave, $u_{P L L}$, in reference to the grid's voltage.

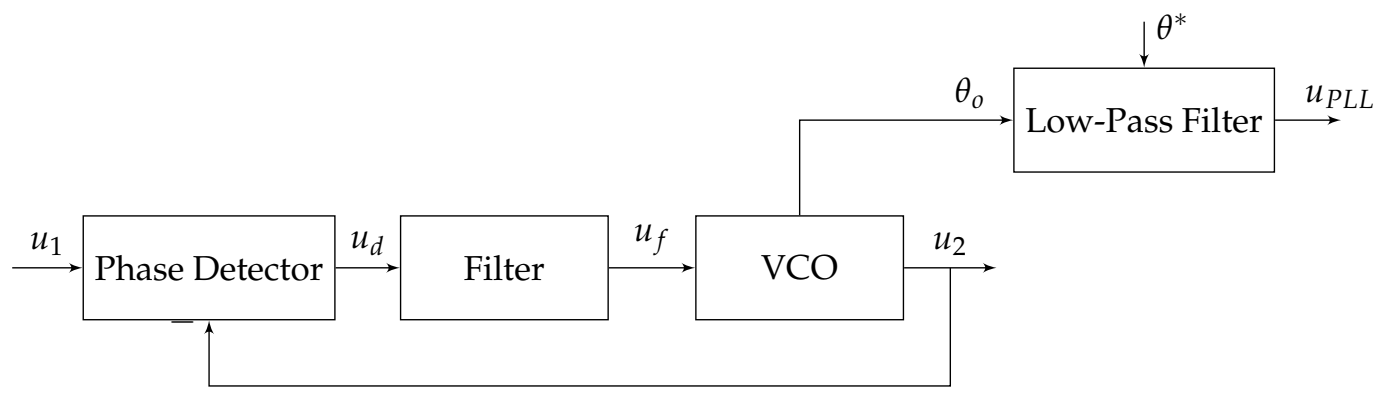

Figure 7. Laboratory implementation in time domain

\section{Discretization}

Discretization is the process to transform a continuous transfer function into a discrete transfer function. There are a few discretization methods [16], in this work, the chosen was the trapezoidal integration, the mathematical formulation is given by (19). Applying this transformation the continuous transfer functions are converted into the discrete domain. The PI filter mathematical formulation in this domain is given in (20), where $T_{S}$ is the sampling time and the $b_{0}, b_{1}$ and $a_{1}$ the discrete filter coefficients.

$$
\begin{gathered}
s \rightarrow \frac{2}{T_{s}} \cdot \frac{z-1}{z+1} \\
\frac{U_{f}(z)}{U_{d}(z)}=\frac{b_{0}+b_{1} \cdot z^{-1}}{1+a_{1} \cdot z^{-1}}=\frac{\frac{\left(T_{s}+2 \cdot \tau_{2}\right)}{2 \cdot \tau_{1}}+\frac{\left(T_{s}-2 \cdot \tau_{2}\right)}{2 \cdot \tau_{1}} \cdot z^{-1}}{1+(-1) \cdot z^{-1}}
\end{gathered}
$$

For the low-pass filter used to attenuate the unwanted harmonics, applying the same transformation, (21) becomes (22), where $\omega_{c}$ is the filter cutoff frequency.

$$
\begin{gathered}
F_{P B}(s)=\frac{\omega_{c}}{s+\omega_{c}} \\
F_{P B}(z)=\frac{b_{0}+b_{1} \cdot z^{-1}}{1+a_{1} \cdot z^{-1}}=\frac{\frac{\left(T_{s} \cdot \omega_{c}\right)}{2+T_{s} \cdot \omega_{c}}+\frac{\left(T_{s} \cdot \omega_{c}\right)}{2+T_{s} \cdot \omega_{c}} \cdot z^{-1}}{1+\left(\frac{-2+T_{s} \cdot \omega_{c}}{2+T_{s} \cdot \omega_{c}}\right) \cdot z^{-1}}
\end{gathered}
$$

The simulation results obtained in the discrete domain are shown on Figure 8. 
Result Without Low-Pass Filter

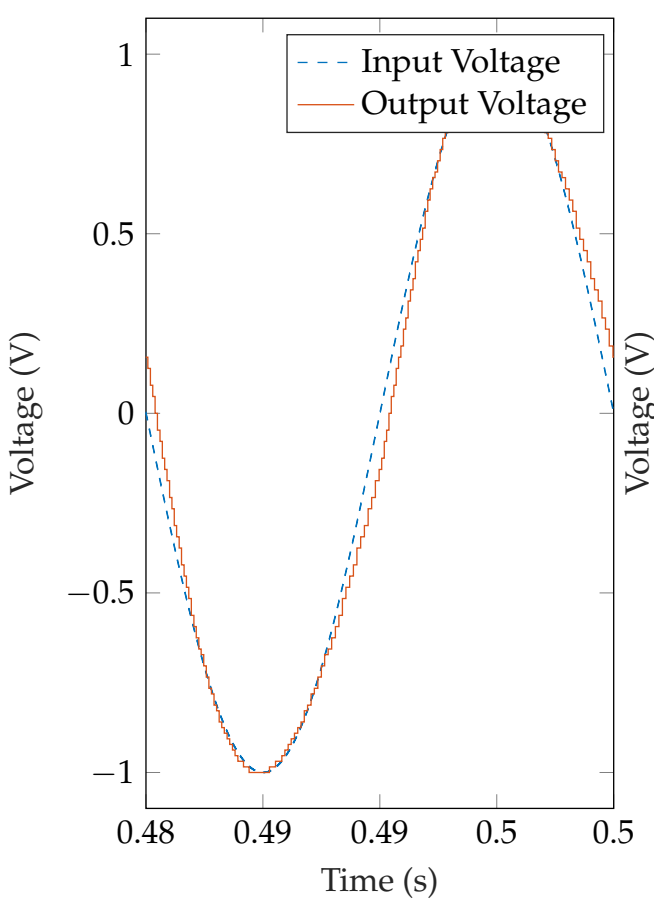

Result With Low-Pass Filter

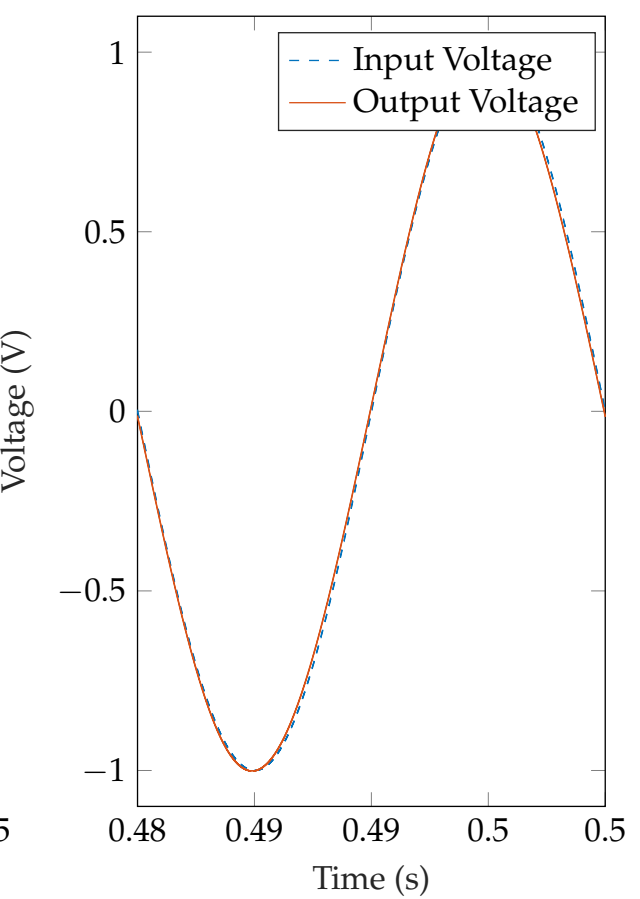

Figure 8. Results for a $50 \mathrm{~Hz}$ sinusoidal input waveform (the input voltage showed represents the grid voltage, the PLL see an square waveform in the input)

\section{Laboratory Implementation}

\subsection{Laboratory Setup}

A $3 \mathrm{~kW}$ experimental prototype of the topology proposed is built. This prototype uses 300A SiC MOSFETs capable of switching at $100 \mathrm{kHz}$, which are a few times higher than the Si-IGBTs equivalent, due to lower switching losses, higher output current capability and significantly lower current de-rating at higher switching frequencies. The energy storage is made using Lithium Iron Phosphate (LiFePO4) batteries at $64 \mathrm{~V}$ of nominal voltage. The objective is to control the power flow between the grid and the battery bank with unity power factor. Figure 10 shows the prototype's power setup, while Figure 11 presents the control electronics setup that uses a low-cost Field Programmable Gate Array (FPGA), Spartan $₫ 3$ (Xilinx Inc., San Jose, CA, USA). Table 3 presents the components specifications used in the prototype.

Table 3. Devices Specifications

\begin{tabular}{|c|c|c|}
\hline Component & Brand & Main Specs \\
\hline SiC Mosfets & Cree & $300 \mathrm{~A}, 1200 \mathrm{~V}, 100 \mathrm{kHz}$ \\
\hline Battery Bank & Calb & $64 \mathrm{~V}, \mathrm{LiFePo} 4$ \\
\hline Transformer & $\mathrm{n} / \mathrm{a}$ & $32 V_{R M S}-230 V_{R M S}, L_{2}=32 \mu \mathrm{H}$ \\
\hline Inductor & Core from Magnetics Inc. & $48 \mu \mathrm{H}$ \\
\hline Capacitors & CDE Cornell Dubilier & $220 \mu \mathrm{F}$ \\
\hline Resistors & $\mathrm{n} / \mathrm{a}$ & $0.45 \Omega$ \\
\hline Current Sensor & LEM & $200 A_{\text {peak }}, \mathrm{DC} 100 \mathrm{kHz}$ \\
\hline FPGA & Xilinx & Spartan $3,125 \mathrm{MHz}$ \\
\hline
\end{tabular}

According to (2) and Table 3, The filter's natural frequency is $2450 \mathrm{~Hz}$ and its Bode Diagram is given on Figure 9. 


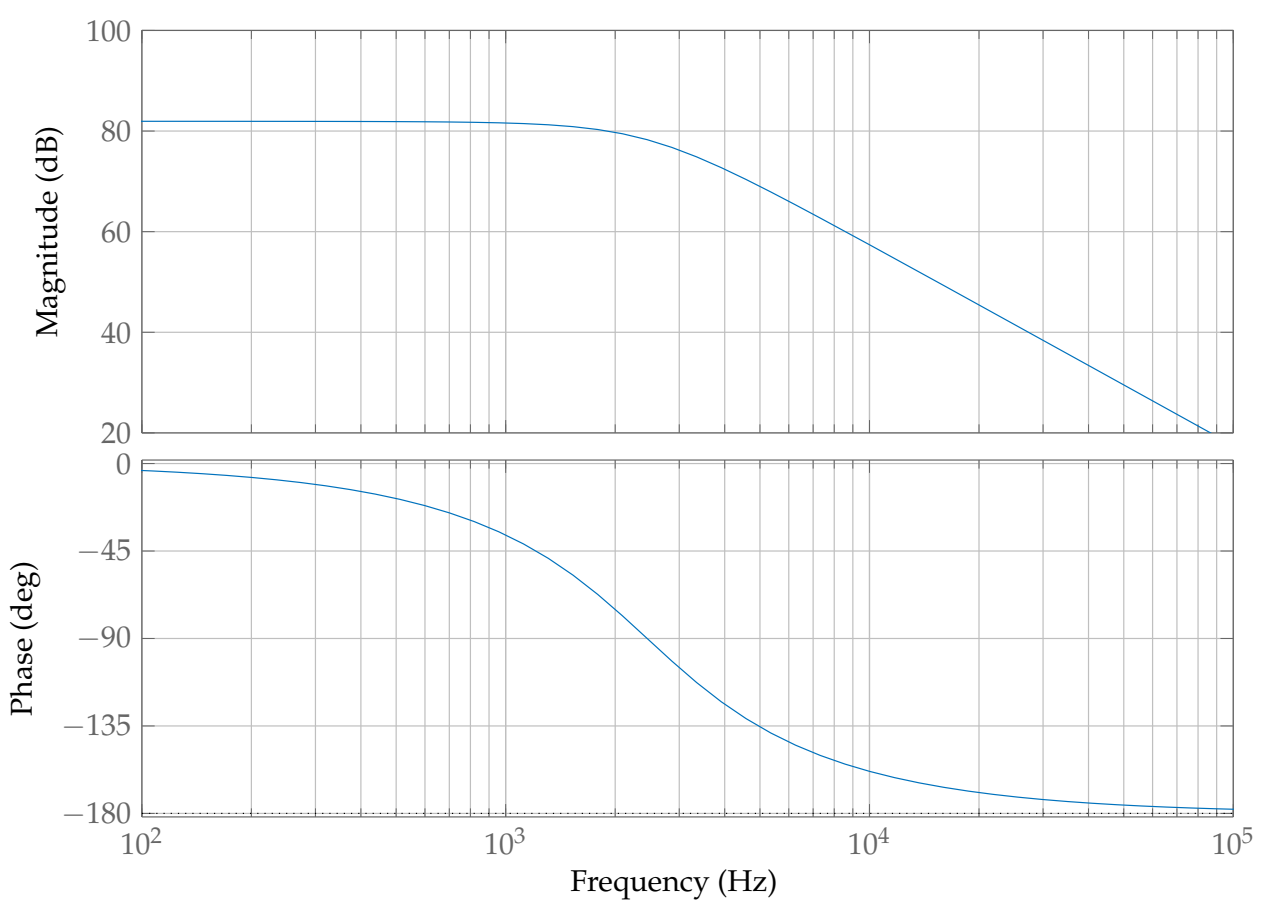

Figure 9. Filter LCL

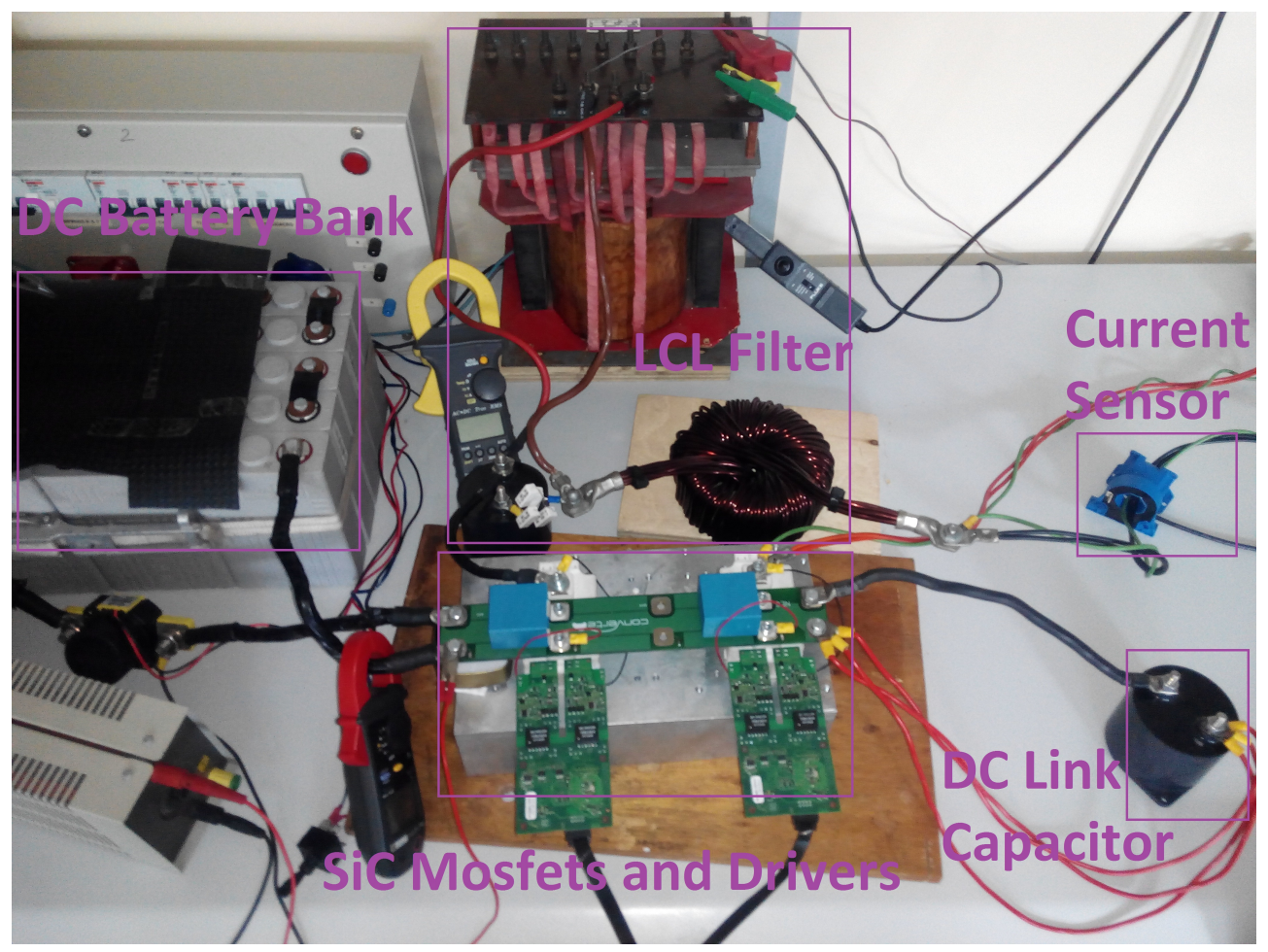

Figure 10. Single-phase SiC bidirectional DC-AC converter power circuit. 


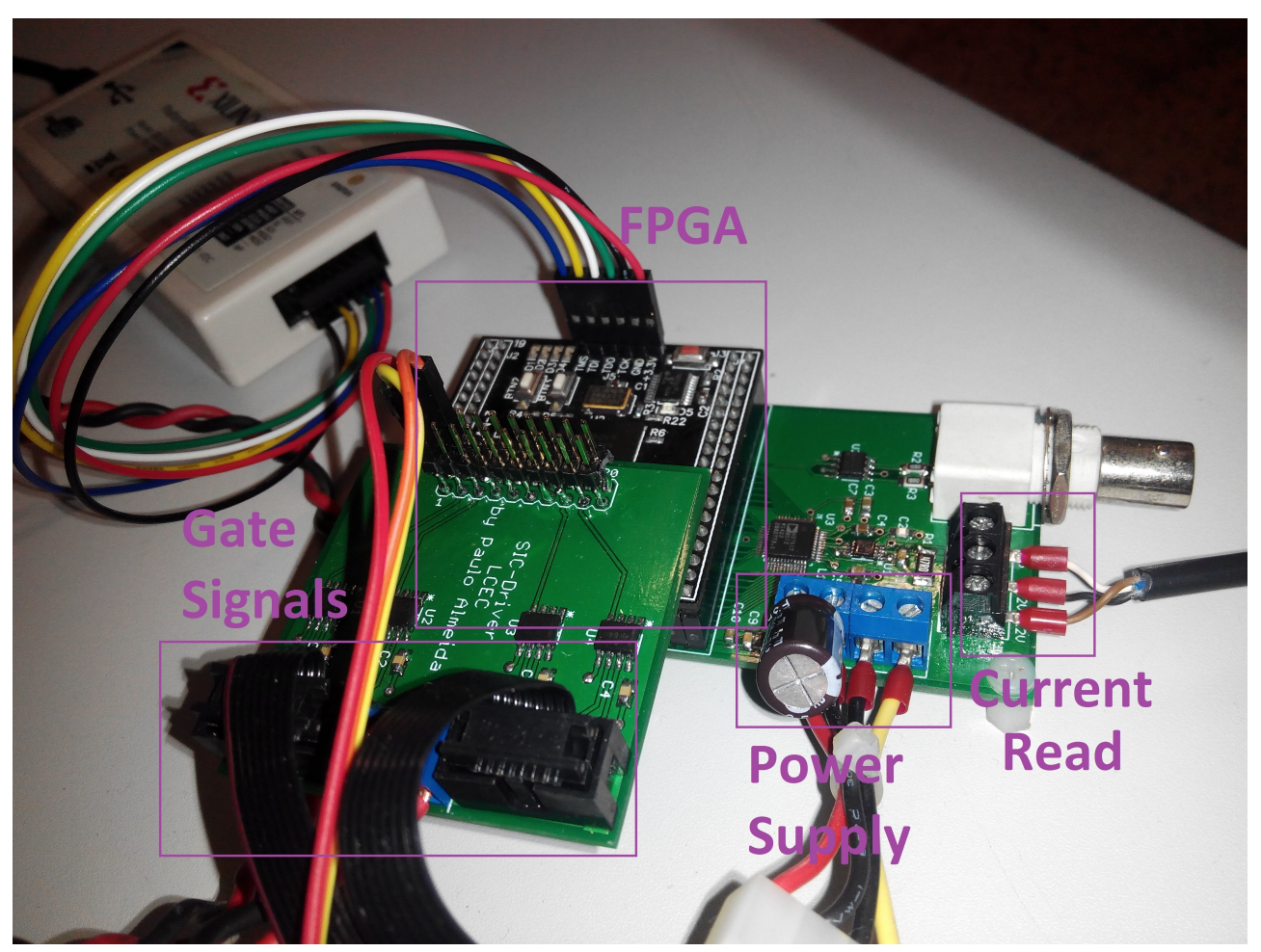

Figure 11. Data acquisition, processing and output generation setup.

\subsection{Phase Locked Loop Implementation}

For this research work, it's was given importance to build a low-cost PLL to synchronize with the grid, but also it was taken into the possibility the use of this PLL to synchronize with different types of waveforms, such as the square and sinusoidal waveforms for future works. Therefore, the multiplier phase detector block was selected and it can be adapted to different waveforms just by adjusting the gains, [13]. Since the integrated circuit used as processor in this work is a FPGA, all the PLL components are implemented in software and just the AC grid voltage was converted into a square wave with the circuit shown on Figure 12. This way the FPGA receives a binary signal that is zero when the grids voltage is positive and one when grids voltage is negative. Note that this signal is isolated by an optocoupler to prevent damaging the FPGA and keep the circuit's cost low. This signal after processing is transformed in signal $u_{1}$ seen in Figure 7.

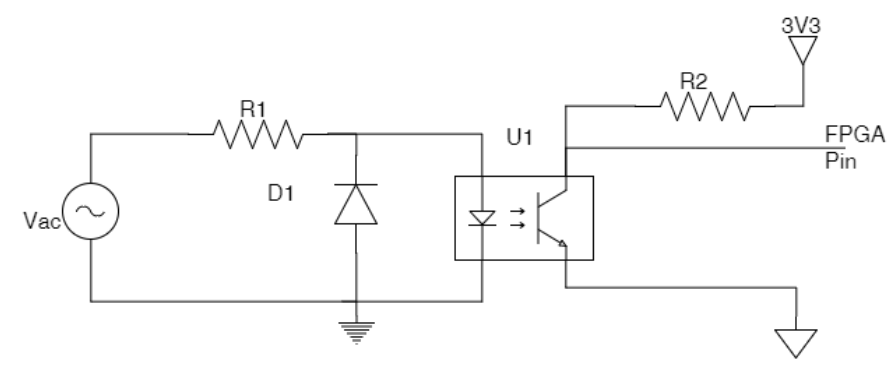

Figure 12. Implemented circuit to detect positive grid voltage.

\subsection{Laboratory Results}

The experimental results were taken at $2 \mathrm{~kW}$ and $3 \mathrm{~kW}$ charging the batteries, see Figures 13 and 14, and then injecting energy on the grid, see Figures 15 and 16. On these experimental results, the current signal is given by channel 4 , while the voltage grid is given by channel 1(at the secondary side of the transformer, $\left.32 V_{R M S}\right)$. Although the current waveform at $2 \mathrm{~kW}$ has a bigger THD than 
operating at $3 \mathrm{~kW}$, that is expected because the current at grid frequency is lower, while the ripple stays the same, the THD still is less than $5 \%$ fulfilling the network requirements.

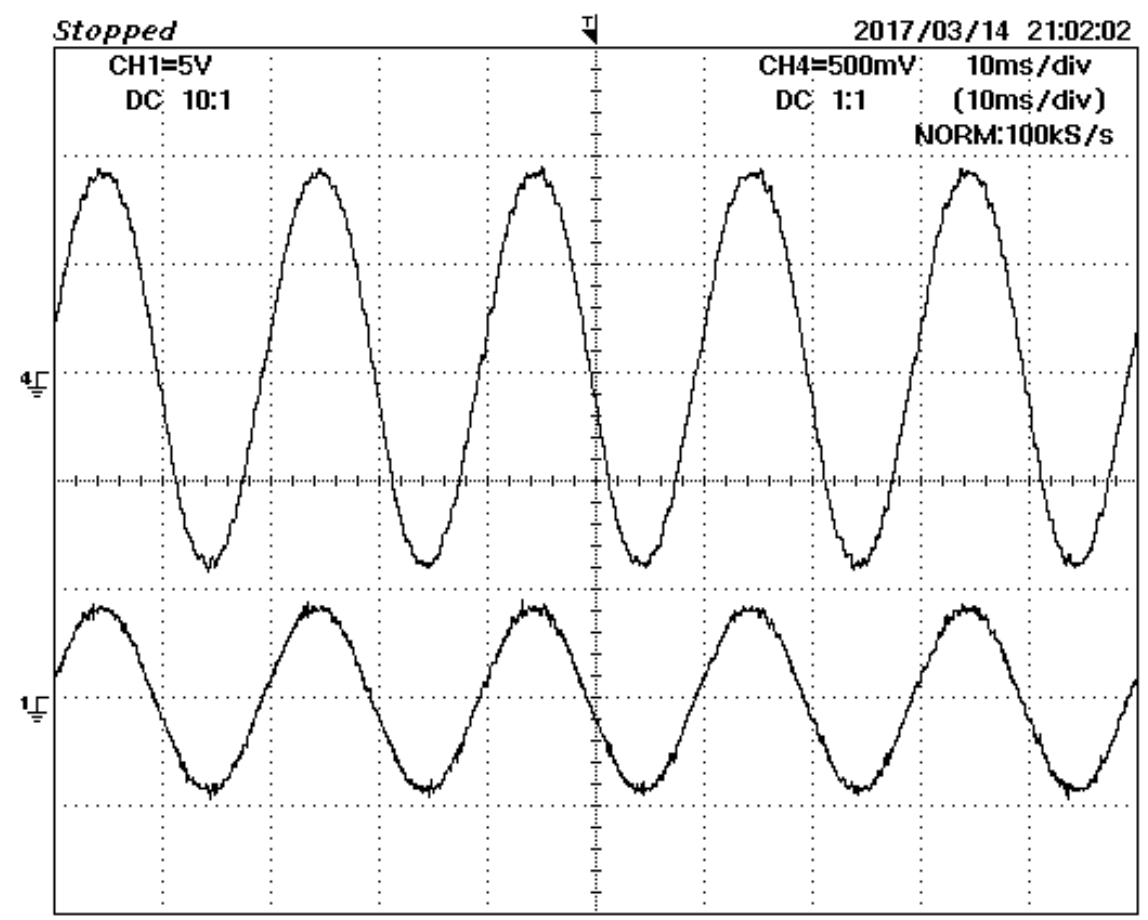

Figure 13. Experimental results charging the batteries at $2 \mathrm{~kW}$ on transformer secundary side ( $32 V_{R M S}$ side). CH1: Grid Voltage (50V/div); CH4: Grid Current (50A/div).

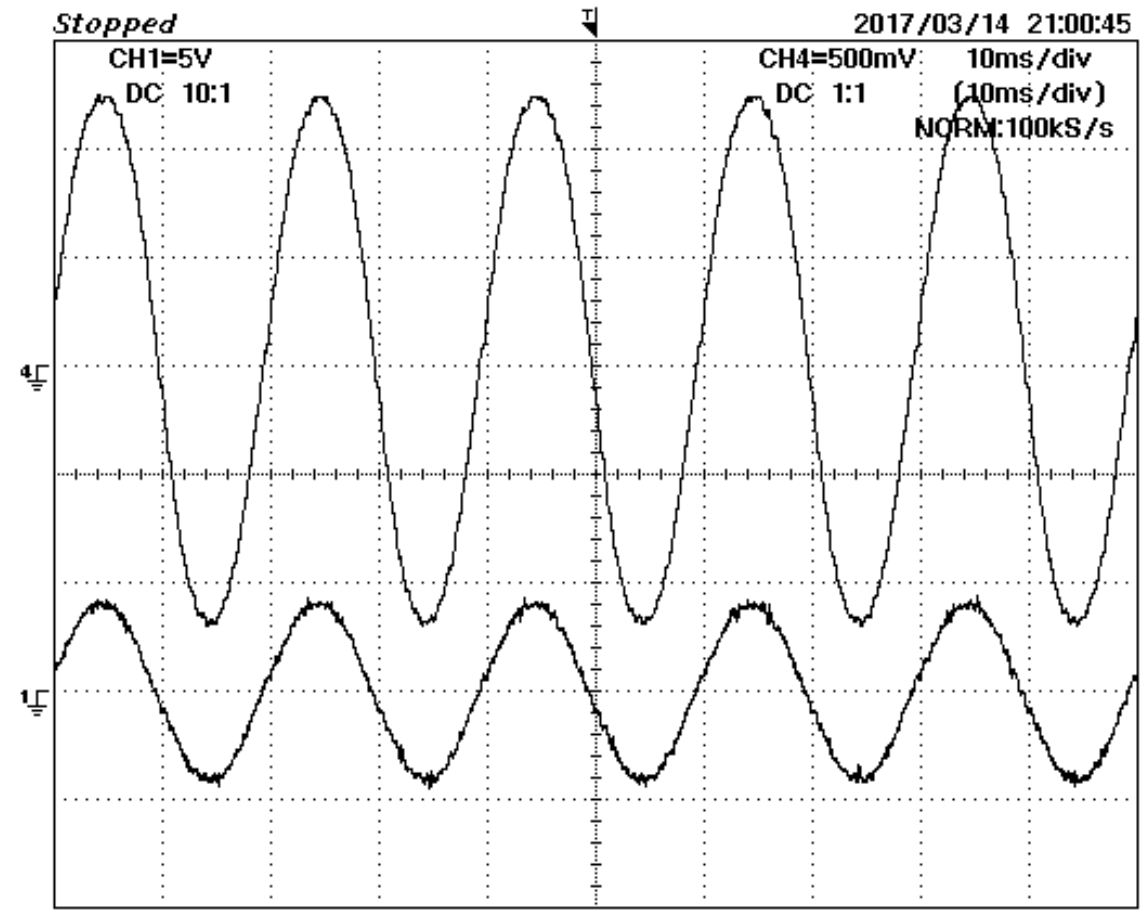

Figure 14. Experimental results charging the batteries at $3 \mathrm{~kW}$ on transformer secundary side $\left(32 V_{R M S}\right.$ side). CH1: Grid Voltage (50V/div); CH4: Grid Current (50A/div). 


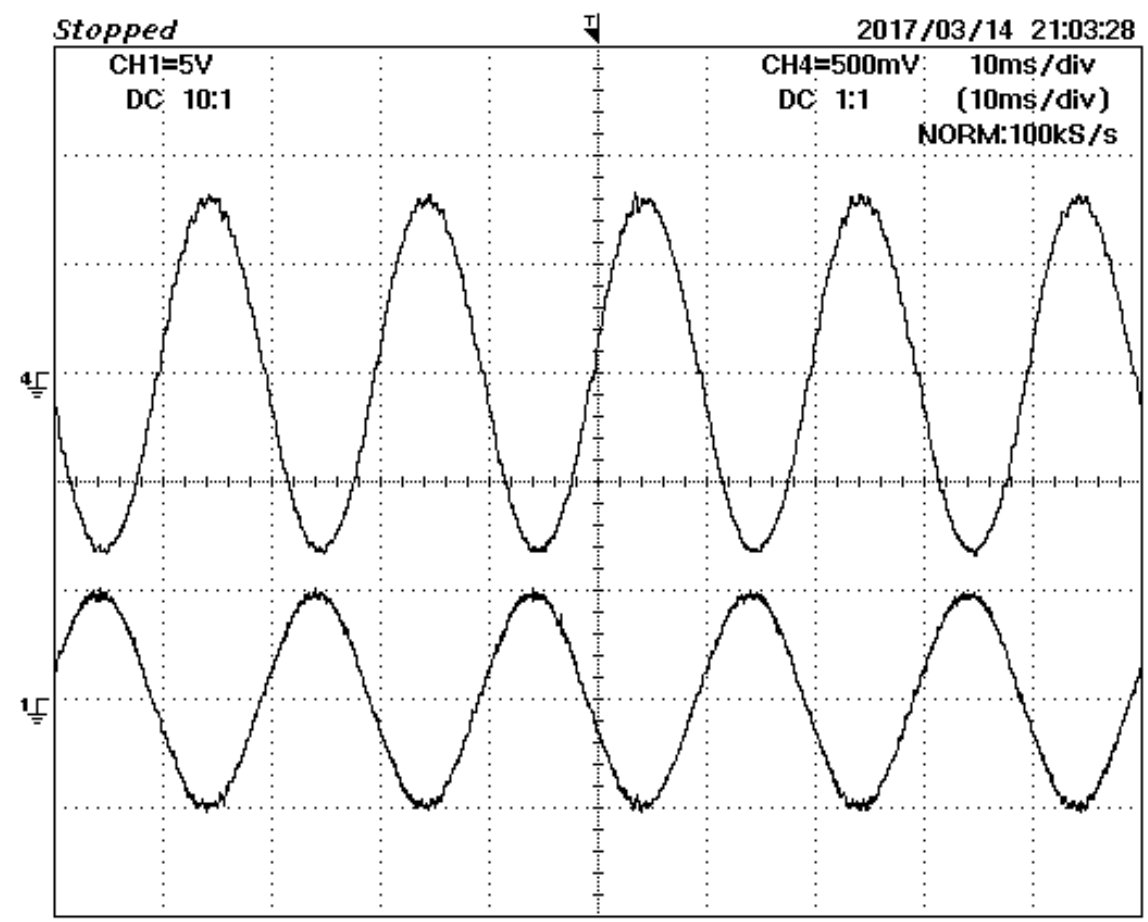

Figure 15. Experimental results injecting $2 \mathrm{~kW}$ on transformer secundary side ( $32 V_{R M S}$ side). $\mathrm{CH} 1$ : Grid Voltage (50V/div); CH4: Grid Current (50A/div).

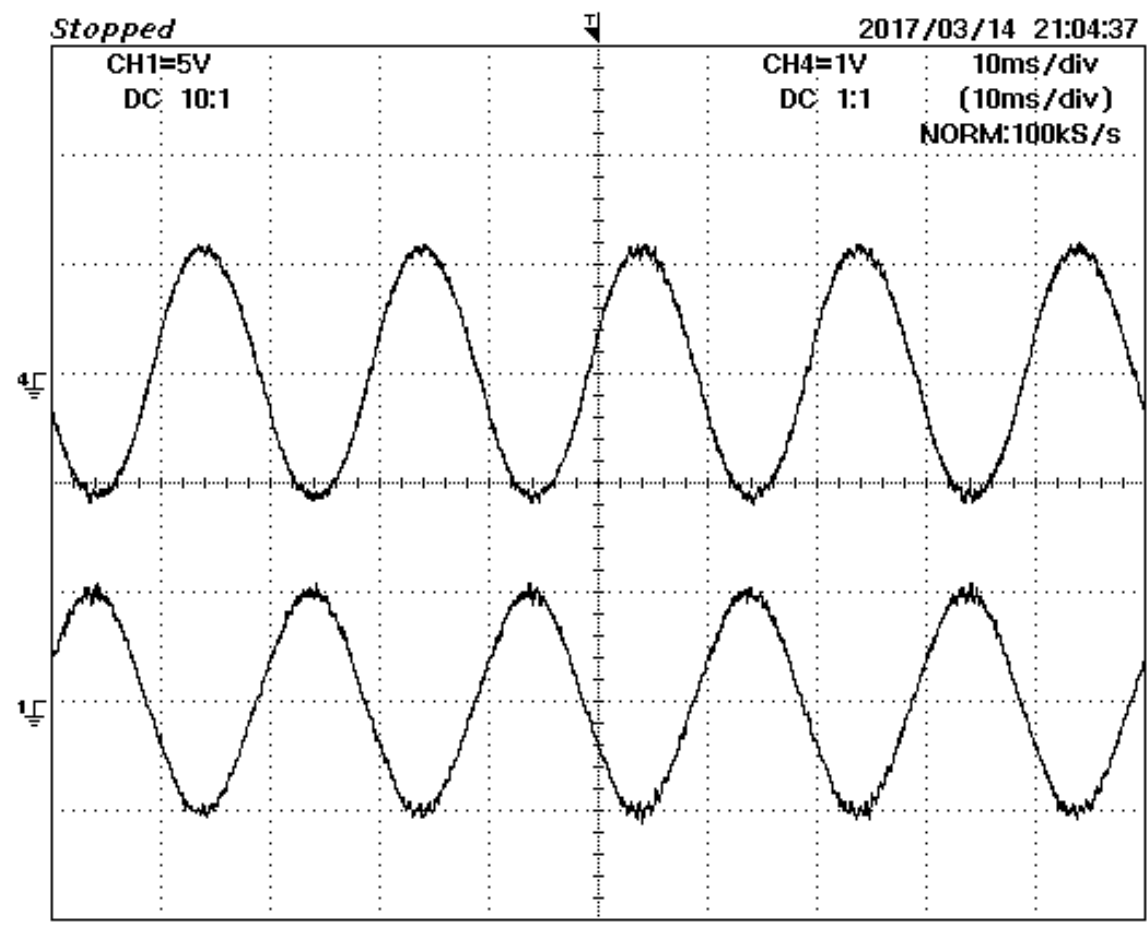

Figure 16. Experimental results injecting $3 \mathrm{~kW}$ on transformer secundary side ( $32 V_{R M S}$ side). $\mathrm{CH} 1$ : Grid Voltage (50V/div); CH4: Grid Current (100A/div). 


\section{Conclusions}

This paper presented the implementation and control of a DC-AC converter with unity power factor with a brief description of inductor design, LCL filter and phase locked loop theory for grid synchronization. It has been introduced the control principles for a variable switching frequency converter and the trade-offs that must be done between system efficiency, filter size and cost while still accomplish the THD limits imposed by legislation. A Converter prototype was built on laboratory, connected to the grid through a $32 V_{R M S}$ to $230 V_{R M S}$ transformer. Laboratory results have been obtained charging the batteries and injecting power on the grid at $2 \mathrm{~kW}$ and $3 \mathrm{~kW}$ which correspond to high currents due to the low voltage level. The measured currents presented THD below 5\%, therefore meeting the standard requirement.

Acknowledgments: Authors thank electrical engineer Paulo Almeida, from ISEL (Polytechnic Institute of Lisbon, Lisboa, Portugal), for the engineering support of the functional prototype assembly. This work was supported by national funds through Fundação para a Ciência e a Tecnologia (FCT) with reference UID/CEC/50021/2013, and in part by EVTV Motor Verks (Cape Girardeau, MO, USA) for the acquisition of the power electronic converter semiconductors.

Author Contributions: Alexandre Bento design and build the LCL filter, build the single-phase SiC bidirectional DC-AC converter, performed the experiments, analyzed the data and wrote the major part of the manuscript. Sérgio André built the PLL circuit, performed its low-level FPGA programming and wrote about the low-cost PLL. Ricardo Luís helped in the high-level FPGA programming and in the experiments. José Fernando Silva supervised the research, providing guidance and key suggestions. All the authors revised and approved the publication of the paper.

Conflicts of Interest: The authors declare no conflict of interest.

\section{References}

1. R. G. Gago, S. F. Pinto, and J. F. Silva. G2V and V2G electric vehicle charger for smart grids. in Smart Cities Conference (ISC2), 2016 IEEE International, 12-15 Sept. 2016.

2. Y. Fan, W. Zhu, Z. Xue, L. Zhang, and Z. Zou. A multi-function conversion technique for vehicle-to-grid applications. Energies 2015, 8, 7638-7653.

3. J. Millan, P. Godignon, X. Perpinya, A. Perez-Tomas, and J. Rebollo. A survey of wide band gap power semiconductor devices. textitIEEE Trans. Power Electron. 2013, 5, 2155-2163.

4. A. Lidow, J. Strydom, M. de Rooij, and D. Reusch. GaN Transistors for Efficient Power Conversion. 2nd ed., California, Wiley, 2015.

5. B. Ozpineci and L. Tolbert. Smaller, faster, tougher. IEEE Spectrum. 2011, vol. 48, 10.

6. J. J. W. Kolar, U. Drofenik, J. Biela, M. L. Heldwein, H. Ertl, T. Friedli, and S. D. Round. PWM converter power density barriers. Power Conversion Conf. - Nagoya, 9-29, 2007.

7. X. Mao, R. Ayyanar, and H. K. Krishnamurthy. Optimal variable switching frequency scheme for reducing switching loss in single-phase inverters based on time-domain ripple analysis. IEEE Trans. Power Electron. 2009, 4, 991-1001.

8. Igoniye, W., Horsfall, O.,Taylor-Harry, S. Experimental Analysis of Exclusive or Phase Locked Loop (PLL). International Journal of Applied and Natural Sciences (IJANS). 2016, 45-52.

9. R. Burkart, H. Uemura, and J. Kolar. Optimal Inductor Design for 3-Phase Voltage-Source PWM Converters Considering Different Magnetic Materials and a Wide Switching Frequency Range. Int. Power Electron. Conf. (ECCE Asia), 891-898, 2014.

10. M. Sippola, Developments for the high frequency power transformer design and implementation. Ph.D. Thesis, Helsinki University of Technology Applied Electronics Laboratory, 2003.

11. A. Bento. Toroid Inductor Development for a SiC DC-DC Converter up to $150 \mathrm{~kW}$, based on Finite Element Method. MSc. Thesis, Instituto Politecnico de Lisboa, 2015.

12. H. C. Sartori, F. Beltrame, M. L. Martins, J. E. Baggio, and J. R. Pinheiro. Evaluation of an Optimal Design for a Single-Phase Boost PFC Converter (CCM) Considering Different Magnetic Materials. 2013 Brazilian Power Electronics Conference. 1304-1310, 2013.

13. Best, R. E. Phase locked loops Design, Simulation and Applications McGraw-Hill Professional, 2007. 
207

208

209

210

211

212

213

14. Golestan, S.,. Freijedo, F. D, Guerrero, J. M. Dynamics Assessment of Advanced Single-Phase PLL Structures. IEEE Trans. Ind. Electron. textbf2013 vol. 60, 2167-2176.

15. Freijedo, F. D., Doval-Gandoy, J., Lopez, O., Cabaleiro, J. Robust phase locked loops optimized for DSP implementation in power quality applications. 2008 34th Annual Conference of IEEE Industrial Electronics. 3052-3057, 2008.

16. Comanescu, M. Influence of the discretization method on the integration accuracy of observers with continuous feedback. 2011 IEEE International Symposium on Industrial Electronics. 625-630, 2011. 